\title{
Functional centrality in graphs
}

\author{
J. A. Rodríguez* \\ Department of Computer Engineering and Mathematics \\ Rovira i Virgili University of Tarragona \\ Av. Països Catalans 26, 43007 Tarragona, Spain \\ E. Estrada ${ }^{\dagger}$ \\ Complex Systems Research Group \\ X-rays Unit, RIAIDT, Edificio CACTUS \\ University of Santiago de Compostela, \\ 15706 Santiago de Compostela, Spain \\ A. Gutiérrez \\ Bayes Inference, S. A. \\ Gran Vía $39,5^{\circ}$ planta \\ 28013 Madrid, Spain
}

\begin{abstract}
In this paper we introduce the functional centrality as a generalization of the subgraph centrality. We propose a general method for characterizing nodes in the graph according to the number of closed walks starting and ending at the node. Closed walks are appropriately weighted according to the topological features that we need to measure.
\end{abstract}

Keywords: Subgraph centrality, graph eigenvalues, complex networks. AMS Subject Classification numbers: 05C65; 05C50; 05C12; 05A20; $15 \mathrm{~A} 42$

*e-mail:juanalberto.rodriguez@urv. cat

†estrada66@yahoo.es

${ }^{\ddagger}$ e-mail:amauri@bayesinf.com 


\section{Introduction}

A kind of local characterization of networks is made numerically by using one of several measures known as centrality [10]. One of the most used centrality measures is the degree centrality, DC [1], which is a fundamental quantity describing the topology of scale-free networks [14]. DC can be interpreted as a measure of immediate influence, as opposed to long-term effect in the network [10]. For instance, if a certain proportion of nodes in the network are infected, those nodes having a direct connection with them will also be infected. However, although a node in a network may be linked to only one node, the risk of infection to the first node remains high if the latter is connected to many others.

There are several other centrality measures that have been introduced and studied for real world networks, in particular for social networks. They account for the different node characteristics that permit them to be ranked in order of importance in the network. Betweenness centrality (BC) characterizes how influential a node is in communicating between node pairs [11]. In other words, BC measures the number of times that a shortest path between nodes $i$ and $j$ travels through a node $k$ whose centrality is being measured. The farness of a vertex is the sum of the lengths of the geodesics to every other vertex. The reciprocal of farness is closeness centrality (CC). The normalized closeness centrality of a vertex is the reciprocal of farness divided by the minimum possible farness expressed as a percentage [1, 10]. This measure is only applicable to connected networks, since the distance between unconnected nodes is undefined. Neither BC nor CC can be related to the network subgraphs in a way that permits them to be considered as measures of node subgraph centrality.

A centrality measure that is not restricted to shortest paths is the eigenvector centrality (EC) [3], which is defined as the principal or dominant eigenvector of the adjacency matrix A representing the connected subgraph or component of the network. It simulates a mechanism in which each node affects all of its neighbors simultaneously [4]. EC cannot be considered as a measure of centrality whereby nodes are ranked according to their participation in different network subgraphs. For instance, in a graph with all nodes having the same degree (a regular graph), all the components of the main eigenvalue are identical [5], even if they participate in different subgraphs. EC is better interpreted as a sort of extended degree centrality which is proportional to the sum of the centralities of the node' neighbors. Consequently, 
a node has high value of EC either if it is connected to many other nodes or if it is connected to others that themselves have high EC [12].
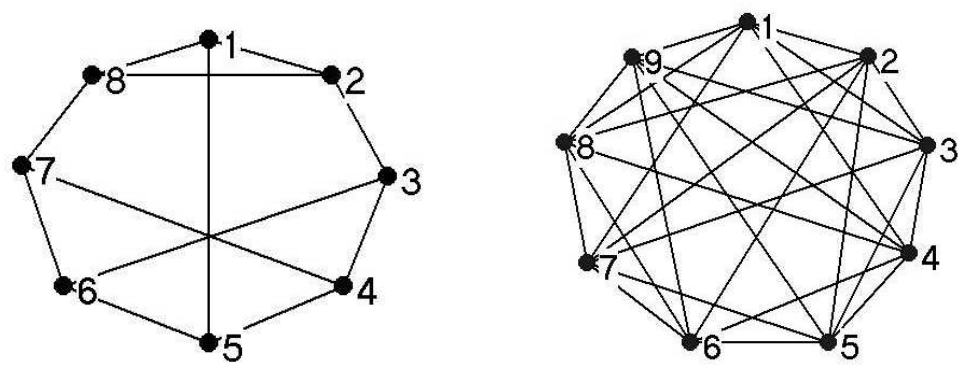

In Figure 1, we illustrate two regular graphs, with eight and nine nodes, and degrees equal to 3 and 6, respectively. In the left hand side graph, nodes $\{1,6,8\}$ are the only ones forming part of a triangle. Vertices $\{4,6\}$ form part of three squares, vertices $\{3,5,7\}$ form part of only two squares and the rest do not form part of any square. The analysis can be obviously extended to larger subgraphs. However, it is evident that there are three groups of distinguishable vertices in the graph, $\{1,2,8\},\{4,6\}$ and $\{3,5,7\}$. These are distinguishable according to their participation in the different subgraphs, although they cannot be distinguished by EC. In the right hand side graph, vertices $\{1,3,5,6,8\}$ take part in 44 of the 100 squares present in the graph, while vertices $\{2,4,7,9\}$ take part in 45 (all vertices take part in the same number of smaller subgraphs; e.g., edges, triangles, connected triples). However, these groups of vertices cannot be distinguished by any of the centrality measures (DC, CC, BC and EC).

A method was proposed in [7] for characterizing nodes in a network according to the number of closed walks starting and ending at the node. Closed walks are appropriately weighted such that their influence on the centrality decreases as the order of the walk increases: the contribution of closed walks of length $k$ to the centrality of the vertex is the number of such closed walks divided by the factorial of $k$. Each closed walk is associated with a connected subgraph, which means that this measure "counts" the times that a node takes part in the different connected subgraphs of the network, with smaller subgraphs having higher importance. Consequently, this measures was called the "subgraph centrality" (SC) for nodes in a network.

In this paper we propose a generalization of the subgraph centrality. We propose a general method for characterizing nodes in the graph according to the number of closed walks starting and ending at the node. Closed walks are 
appropriately weighted according to the topological features that we need to measure.

\section{Functional centrality}

The following well-known result will be useful in the spectral study of centralities.

Theorem 1. [2] Let $v_{i}$ and $v_{j}$ be vertices of a graph $\Gamma$. Let $\boldsymbol{A}$ be the adjacency matrix of $\Gamma$. Then, the number of walks of length $k$ in $\Gamma$, from $v_{i}$ to $v_{j}$, is the entry in position $(i, j)$ of the matrix $\boldsymbol{A}^{k}$.

Since the adjacency matrix, $\mathbf{A}$, of $\Gamma$ is a symmetric matrix with real entries, there exists an orthogonal matrix $U=\left(u_{i j}\right)$ such that $\mathbf{A}=U D U^{T}$ where $D=\operatorname{diag}\left(\lambda_{1}, \lambda_{2}, \ldots, \lambda_{n}\right)$ whose diagonal entries are the eigenvalues of A, and the columns of $U$ are the corresponding eigenvectors that form an orthogonal basis of the Euclidean space $\mathbb{R}^{n}$. Hereafter we will denote by $\lambda$ the main eigenvalue of $\mathbf{A}$ and the $j$ th column of $U$ will be denoted by $U_{j}$. It must be emphasized that, if the graph $\Gamma$ is connected, then the symmetric and non-negative matrix $\mathbf{A}$ is irreducible. As a consequence, the main eigenvalue of $\mathbf{A}$ has a positive eigenvector of multiplicity one. This fact facilitates the use of the main eigenvector as a measure of centrality [3]. It follows that walks of length $k$ in $\Gamma$, from $v_{i}$ to $v_{j}$, are

$$
\mu_{k}(i j)=\left(\mathbf{A}^{k}\right)_{i j}=\sum_{s=1}^{n} u_{i s} u_{j s} \lambda_{s}^{k}
$$

Moreover, the number of closed walks of length $k$ starting and ending on vertex $v_{i}$ in $\Gamma$ is given by the local spectral moments $\mu_{k}(i)$, which are simply defined as the $i$ th diagonal entry of the $k$ th power of the adjacency matrix, A:

$$
\mu_{k}(i)=\left(\mathbf{A}^{k}\right)_{i i}=\sum_{s=1}^{n}\left(u_{i s}\right)^{2} \lambda_{s}^{k} .
$$

Let $\lambda$ be the spectral radius of $\Gamma$. Let $f$ be a function, $f: \mathbb{R} \mapsto \mathbb{R}$, whose Taylor series is $f(x)=\sum_{k=0}^{\infty} a_{k} x^{k},|x|<\lambda_{*}$, where $\lambda_{*}>\lambda$. We define the 
functional centrality, $C_{f}(i)$, as

$$
C_{f}(i):=\sum_{j=0}^{\infty} a_{j} \mu_{j}(i) .
$$

In this case, the number of closed walks of length $l$ is weighted by $a_{l}$. Thus, we can select the function $f$ according to the topological features that we need to measure. Some interesting cases will be explained in the next sections.

For any $v_{i} \in V$ we denote by $\ell^{i}(\mathbb{N})$ the space of real sequences $y=\left(y_{j}\right)_{j=0}^{\infty}$ such that

$$
\sum_{j=0}^{\infty} y_{j} \mu_{j}(i)<\infty
$$

Theorem 2. Let $\Gamma=(V, E)$ be a simple graph of order $n$. Let $\lambda_{1} \geq \lambda_{2} \geq$ $\cdots \geq \lambda_{n}$ be the eigenvalues of $\Gamma$ and let $U=\left(u_{i j}\right)$ denotes an orthogonal matrix whose columns are the corresponding eigenvectors. Let $\lambda_{*}>\lambda_{1}$ and let $f$ be a real function such that $f(x)=\sum_{k=0}^{\infty} a_{k} x^{k}$, for $|x|<\lambda_{*}$. Then for each $v_{i} \in V, a=\left(a_{0}, a_{1}, \ldots, a_{k}, \ldots\right) \in \ell^{i}(\mathbb{N})$ and the functional centrality is

$$
C_{f}(i)=\sum_{j=1}^{n}\left(u_{i j}\right)^{2} f\left(\lambda_{j}\right) .
$$

Proof. By the definition of $C_{f}(i)$ and (2), we obtain

$$
C_{f}(i)=\sum_{k=0}^{\infty} a_{k}\left(\sum_{j=1}^{n} \lambda_{j}^{k}\left(u_{i j}\right)^{2}\right) .
$$

On the other hand, series (4) is obtained by adding term by term the following 
convergent series:

$$
\begin{gathered}
\left(u_{i 1}\right)^{2} \sum_{k=0}^{\infty} a_{k} \lambda_{1}^{k}=\left(u_{i 1}\right)^{2} f\left(\lambda_{1}\right) \\
\left(u_{i 2}\right)^{2} \sum_{k=0}^{\infty} a_{k} \lambda_{2}^{k}=\left(u_{i 2}\right)^{2} f\left(\lambda_{2}\right) \\
\vdots \\
\left(u_{i n}\right)^{2} \sum_{k=0}^{\infty} a_{k} \lambda_{n}^{k}=\left(u_{i n}\right)^{2} f\left(\lambda_{n}\right) .
\end{gathered}
$$

Thus, series (4) converges to $\sum_{j=1}^{n}\left(u_{i j}\right)^{2} f\left(\lambda_{j}\right)$.

Theorem 3. Let $\Gamma=(V, E)$ be a nontrivial simple graph of order $n$ and spectral radius $\lambda$. Let $\lambda_{*}>\lambda$ and let $f$ be a real function such that $f(x)=$ $\sum_{k=0}^{\infty} a_{k} x^{k}$, for $|x|<\lambda_{*}$, where $a_{l} \geq 0$ for all $l$. Then for each $v_{i} \in V$,

$$
C_{f}(i) \leq \frac{1}{n}[f(n-1)+(n-1) f(-1)] .
$$

The equality holds if and only if $\Gamma$ is the complete graph.

Proof. Since $\Gamma$ is nontrivial, let $x$ be an edge of $\Gamma$. Let $\Gamma-x$ be the graph obtained by removing $x$ from $\Gamma$. Then the number of closed walks of length $k$ in $\Gamma-x$ is equal to the number of closed walks of length $k$ in $\Gamma$ minus the number of closed walks of length $k$ in $\Gamma$ containing $x$. Consequently, as $a_{l} \geq 0$ for all $l$, for all $v_{i} \in V, C_{f}(i)$ in $\Gamma-x$ is lower than or equal to $C_{f}(i)$ in $\Gamma$. In closing, the maximum $C_{f}(i)$ is attained if and only if $\Gamma$ is the complete graph $\Gamma=K_{n}$. We now compute $C_{f}(i)$ in $K_{n}$. The eigenvalues of $\Gamma$ are $n-1$ and -1 (with multiplicity 1 and $n-1$ ). By spectral decomposition of unit vector $e_{i} \in \mathbb{R}^{n}$,

$$
e_{i}=u_{i 1} U_{1}+\sum_{j=2}^{n} u_{i j} U_{j}
$$

we obtain

$$
1=\left\|e_{i}\right\|^{2}=\frac{1}{n}+\sum_{j=2}^{n}\left(u_{i j}\right)^{2}
$$


Therefore, we have

$$
\begin{aligned}
C_{f}(i) & =\sum_{j=1}^{n}\left(u_{i j}\right)^{2} f\left(\lambda_{j}\right) \\
& =\frac{1}{n} f(n-1)+f(-1) \sum_{j=2}^{n}\left(u_{i j}\right)^{2} \\
& =\frac{1}{n}[f(n-1)+(n-1) f(-1)] .
\end{aligned}
$$

\subsection{Subgraph Centrality}

The subgraph centrality, [7], is defined as

$$
C_{S}(i):=\sum_{k=0}^{\infty} \frac{\mu_{k}(i)}{k !}
$$

In this case, closed walks are appropriately weighted such that their influence on the centrality decreases as the order of the walk increases. Each closed walk is associated with a connected subgraph, which means that this measure "counts" the times that a node takes part in the different connected subgraphs of the graph, with smaller subgraphs having higher importance.

Let $\lambda$ be the main eigenvalue of $\mathbf{A}$. For any non-negative integer $k$ and any $v_{i} \in V(\Gamma)$ we have $\mu_{k}(i) \leq \lambda^{k}$ and thus series (5) - whose terms are nonnegative - converges:

$$
\sum_{k=0}^{\infty} \frac{\mu_{k}(i)}{k !} \leq \sum_{k=0}^{\infty} \frac{\lambda^{k}}{k !}=e^{\lambda}
$$

Thus, $a=\left(1,1, \frac{1}{2 !}, \frac{1}{3 !}, \ldots, \frac{1}{k !}, \ldots\right) \in \ell^{i}(\mathbb{N}), \forall v_{i} \in V(\Gamma)$. Obviously, in this case, $f(x)=e^{x}$ and the subgraph centrality of $v_{i} \in V$ is $C_{S}(i)=\sum_{j=1}^{n}\left(u_{i j}\right)^{2} e^{\lambda_{j}}$.

The reader is referred to [7] for a detailed study on subgraph centrality and its applications to complex networks. 


\subsection{Monomial centrality}

Suppose that the problem is to characterize nodes in the graph according to the number of closed walks of length $k$ containing the node, i.e., the local spectral moments. In this case we take the function $f(x)=x^{k}$ and, in consequence, we call this centrality monomial centrality. Thus, the monomial centrality $C_{k}(i)$, is defined as

$$
C_{k}(i):=\mu_{k}(i) .
$$

For instance, if $k=2$, we obtain the degree centrality.

\subsection{Functional centrality of radius $k$}

Suppose that the problem is to characterize nodes in the graph according to the number of closed walks of length lower than or equal to $k$ containing the node. In this case we take the function $f$ as a polynomial of degree $k, f_{k}(x)=a_{0}+a_{1} x+a_{2} x^{2}+\cdots+a_{k} x^{k}$, where the coefficients are taking according to the importance of the participation of the node in the closed walks of a given length. For instance, if we consider that the contribution of closed walks of length $1 \leq l \leq k$ to the centrality is inversely proportional to $l$, then we take $a_{l}=\frac{1}{l}$. Thus, $C_{f_{k}}(i)=\sum_{j=1}^{n}\left(u_{i j}\right)^{2}\left(1+\lambda_{j}+\frac{\lambda_{j}^{2}}{2}+\cdots+\frac{\lambda_{j}^{k}}{k}\right)$.

\subsection{Odd and even centralities}

It is well-known that there are graphs that do not have odd closed-walks. i.e., the bipartite graphs. On the other hand, it would be of some interest to characterize nodes in the graphs according to the number of closed walks of odd (even) length containing the node. In such a case we can take an odd (even) function. For instance, the odd subgraph centrality is defined as

$$
C_{S_{\text {odd }}}(i):=\frac{\mu_{1}(i)}{1 !}+\frac{\mu_{3}(i)}{3 !}+\frac{\mu_{5}(i)}{5 !}+\cdots
$$

Hence, in this case, $f(x)=\sinh (x)$ and $C_{S_{\text {odd }}}(i)=\sum_{j=1}^{n}\left(u_{i j}\right)^{2} \sinh \left(\lambda_{j}\right)$. Analogously, the even subgraph centrality is defined as

$$
C_{S_{\text {even }}}(i):=\frac{\mu_{0}(i)}{0 !}+\frac{\mu_{2}(i)}{2 !}+\frac{\mu_{4}(i)}{4 !}+\cdots
$$


Thus, $f(x)=\cosh (x)$ and $C_{S_{\text {even }}}(i)=\sum_{j=1}^{n}\left(u_{i j}\right)^{2} \cosh \left(\lambda_{j}\right)$.

As we will show in Section 3, the above centralities are of singular importance in the study of bipartivity.

\subsection{Example}

In order to show the differences in the orders imposed by different functional centralities, we have selected the left hand side graph of Figure 1 and the following centrality measures.

- Monomial centrality, $C_{3}: f(x)=x^{3}$.

- Functional centrality of radius $3, C_{f_{3}}: f(x)=1+x+\frac{x^{2}}{2}+\frac{x^{3}}{3}$.

- Functional centrality of radius $4, C_{f_{4}}: f(x)=1+x+\frac{x^{2}}{2}+\frac{x^{3}}{3}+\frac{x^{4}}{4}$.

- Odd centrality, $C_{S_{\text {odd }}}: f(x)=\sinh (x)$.

- Subgraph centrality, $C_{s}: f(x)=e^{x}$.

\begin{tabular}{|c|c|c|c|c|c|c|c|c|}
\hline & $\mathbf{1}$ & $\mathbf{2}$ & $\mathbf{3}$ & $\mathbf{4}$ & $\mathbf{5}$ & $\mathbf{6}$ & $\mathbf{7}$ & $\mathbf{8}$ \\
\hline$C_{3}$ & 2 & 2 & 0 & 0 & 0 & 0 & 0 & 2 \\
\hline$C_{f_{3}}$ & 3.16 & 3.16 & 2.5 & 2.5 & 2.5 & 2.5 & 2.5 & 3.16 \\
\hline$C_{f_{4}}$ & 6.92 & 6.92 & 7.25 & 7.75 & 7.25 & 7.75 & 7.25 & 6.92 \\
\hline$C_{S_{\text {odd }}}$ & 0.608 & 0.608 & 0.117 & 0.075 & 0.117 & 0.075 & 0.117 & 0.608 \\
\hline$C_{s}$ & 3.9 & 3.9 & 3.63 & 3.7 & 3.63 & 3.7 & 3.63 & 3.9 \\
\hline
\end{tabular}

\section{Functional centralization}

By (1) and (2) we have that the number $w_{k}$ of walks of length $k$ in $\Gamma$ is given by

$$
w_{k}=\sum_{i, j} \mu_{k}(i j)=\sum_{s=1}^{n}\left(\sum_{i=1}^{n} u_{i s}\right)^{2} \lambda_{s}^{k},
$$

and the number $\theta_{k}$ of closed-walks of length $k$ in $\Gamma$ is given by the trace of $\mathbf{A}^{k}$ :

$$
\theta_{k}=\sum_{i=1}^{n} C_{k}(i)=\sum_{s=1}^{n} \lambda_{s}^{k}
$$


Let $\lambda$ be the spectral radius of $\Gamma$. Let $f$ be a function, $f: \mathbb{R} \mapsto \mathbb{R}$, whose Taylor series is $f(x)=\sum_{k=0}^{\infty} a_{k} x^{k},|x|<\lambda_{*}$, where $\lambda_{*}>\lambda$. We define the functional centralization, $C_{f}(\Gamma)$, as

$$
C_{f}(\Gamma):=\sum_{j=0}^{\infty} a_{j} \theta_{j}
$$

Hence, an analytical expression for $C_{f}(\Gamma)$, which depends only on the eigenvalues and order of the graph, can be obtained by using a procedure analogous to that described in the proof of Theorem 2.

Theorem 4. Let $\Gamma=(V, E)$ be a simple graph of order $n$ and let $\lambda_{1} \geq \lambda_{2} \geq$ $\cdots \geq \lambda_{n}$ be the eigenvalues of $\Gamma$. Let $\lambda_{*}>\lambda_{1}$ and let $f$ be a real function such that $f(x)=\sum_{k=0}^{\infty} a_{k} x^{k}$, for $|x|<\lambda_{*}$. Then the functional centralization is

$$
C_{f}(\Gamma)=\sum_{j=1}^{n} f\left(\lambda_{j}\right)=\sum_{i=1}^{n} C_{f}(i)
$$

For instance, taking $f(x)=x^{k}$ we obtain the $k$-spectral moment of $\Gamma$,

$$
C_{k}(\Gamma):=\theta_{k}=\sum_{s=1}^{n} \lambda_{s}^{k}
$$

This centralization only measures the number of closed-walks of length $k$ in $\Gamma$. For instance, if $k=2$, then $C_{2}(G)=2 m$, where $m$ denotes the size of $G$.

Taking $f(x)=e^{x}$ we obtain the subgraph centralization:

$$
C_{S}(\Gamma):=\sum_{k=0}^{\infty} \frac{\theta_{k}}{k !} .
$$

That is,

$$
C_{S}(\Gamma)=\sum_{i=1}^{n} e^{\lambda_{i}}
$$

Taking $f(x)=\sinh (x)$ in Theorem 4 we obtain the odd subgraph centralization of $\Gamma$

$$
C_{S_{\text {odd }}}(\Gamma)=\sum_{i=1}^{n} \sinh \left(\lambda_{i}\right) .
$$


Analogously, the even subgraph centralization is

$$
C_{S_{\text {even }}}(\Gamma)=\sum_{i=1}^{n} \cosh \left(\lambda_{i}\right) .
$$

Both subgraph centralization and even centralization, were used to obtain measures of bipartivity in graphs [8]: the proportion of even closed walks to the total number of closed walks is a measure of the graph bipartivity. Thus, we measures the bipartivity of a graph $\Gamma$ by

$$
\beta(\Gamma)=\frac{\sum_{i=1}^{n} \cosh \left(\lambda_{i}\right)}{\sum_{i=1}^{n} e^{\lambda_{i}}} .
$$

It is evident that $\beta(\Gamma) \leq 1$ and $\beta(\Gamma)=1$ if and only if $\Gamma$ is bipartite. Moreover, as $e^{\lambda_{i}}=\sinh \left(\lambda_{i}\right)+\cosh \left(\lambda_{i}\right)$ and $\sinh \left(\lambda_{i}\right)<\cosh \left(\lambda_{i}\right), \forall i$, we have $\frac{1}{2}<\beta(\Gamma) \leq 1$. The lower bound is reached for the least possible bipartite graph with $n$ nodes, which is the complete graph $K_{n}$. As the eigenvalues of $K_{n}$ are $n-1$ and -1 (with multiplicity $n-1$ ), then $\beta(\Gamma) \rightarrow \frac{1}{2}$ when $n \rightarrow \infty$ in $K_{n}$.

The contribution of vertex $v_{i}$ to graph bipartivity, $\beta(i)$, can be obtained by using the appropriate functional centrality of $v_{i}$. That is,

$$
\beta(i)=\frac{\sum_{j=1}^{n}\left(u_{i j}\right)^{2} \cosh \left(\lambda_{j}\right)}{\sum_{j=1}^{n}\left(u_{i j}\right)^{2} e^{\lambda_{j}}} .
$$

The reader is referred to [8] for a more detailed study on bipartivity and its applications. Moreover, an application of our bipartivity measures to the study of fullerene graphs can be found in [6].

\section{Functional centrality in hypergraphs}

Let $\mathcal{H}$ be a simple hypergraph of order $n$. The adjacency matrix, $\mathbf{A}(\mathcal{H})$, of the hypergraph $\mathcal{H}=(V, E)$ is a square symmetric matrix whose entries 
$a_{i j}$ are the number of hyper-edges that contain both nodes $v_{i}$ and $v_{j}$ : the diagonal entries of $\mathbf{A}(\mathcal{H})$ are zero. This can be obtained from the incidence matrix of $\mathcal{H}$ as follows:

$$
\mathbf{A}(\mathcal{H})=\mathbf{E E}^{T}-\mathbf{D}
$$

where $\mathbf{E}^{T}$ is the transpose of the incidence matrix and $\mathbf{D}$ is the diagonal matrix whose diagonal entries are the degrees of the vertices. More formally, $\mathbf{A}(\mathcal{H})$ is a $n \times n$ matrix with diagonal entries $a_{i i}=0$, for $v_{i} \in V$, and off-diagonal entries

$$
a_{i j}=\left|\left\{E_{k} \in E(\mathcal{H}):\left\{v_{i}, v_{j}\right\} \subset E_{k}\right\}\right|, \quad \text { for } \quad v_{i}, v_{j} \in V(\mathcal{H}), \quad i \neq j .
$$

Since $\mathbf{A}(\mathcal{H})$ is symmetric, and its entries are non-negative integers, it may be viewed as the adjacency matrix of a multigraph $G^{\prime}$, i.e., a graph having multiple links between nodes, called the associated graph of $\mathcal{H}=(V, E)$.

It must be emphasized that, if the hypergraph $\mathcal{H}$ is connected, then the symmetric and non-negative matrix $\mathbf{A}(\mathcal{H})$ is irreducible. As a consequence, the main eigenvalue of $\mathbf{A}(\mathcal{H})$ has a positive eigenvector of multiplicity one. This fact facilitates the extension, to the case of hypergraphs, of the use of the main eigenvector as a measure of centrality.

The following result will be useful in extending the definition of functional centrality to hypergraphs.

Theorem 5. [13] Let $v_{i}$ and $v_{j}$ be vertices of a hypergraph $\mathcal{H}$. Let $\boldsymbol{A}(\mathcal{H})$ be the adjacency matrix of $\mathcal{H}$. Then, the number of walks of length $k$ in $\mathcal{H}$, from $v_{i}$ to $v_{j}$, is the entry in position $(i, j)$ of the matrix $(\boldsymbol{A}(\mathcal{H}))^{k}$.

So, the spectral study of centralities is completely analogous to the previous one for graphs.

Theorem 6. Let $\mathcal{H}$ be a simple hypergraph of order $n$. Let $\lambda_{1} \geq \lambda_{2} \geq \cdots \geq$ $\lambda_{n}$ be the eigenvalues of $G_{\mathcal{H}}$ and let $\boldsymbol{U}=\left(\boldsymbol{u}_{i j}\right)$ denote an orthogonal matrix whose columns are the corresponding eigenvectors. Let $\lambda_{*}>\lambda_{1}$ and let $f$ be a real function such that $f(x)=\sum_{k=0}^{\infty} a_{k} x^{k}$, for $|x|<\lambda_{*}$. Then for each $v_{i} \in V(\mathcal{H}), a=\left(a_{0}, a_{1}, \ldots, a_{k}, \ldots\right) \in \ell^{i}(\mathbb{N})$ and the functional centrality is

$$
C_{f}(i)=\sum_{j=1}^{n}\left(\boldsymbol{u}_{i j}\right)^{2} f\left(\lambda_{j}\right)
$$


Proof. The proof is basically as in Theorem 2.

The particular case of subhypergraph centrality, i.e., $f(x)=e^{x}$, was analyzed in [9].

\section{References}

[1] A. Albert, H. Jeong, and A.L. Barabási, Diameter of the world wide web. Nature. 401 (1999) 130-131.

[2] N. Biggs, Algebraic Graph Theory, Cambridge University Press, Cambridge, 1993.

[3] P. Bonacich, Factoring and weighting approaches to status scores and clique identification. J. Math. Sociol. 2 (1972) 113-120.

[4] P. Bonacich, Power and centrality: A family of measures, Am. J. Sociol. 92 (1987) 1170-1182.

[5] D. Cvetković, P. Rowlinson and S. Simic,Eigenspaces of Graphs (Cambridge Univ. Press, Cambridge, UK 1997.

[6] Tomislav Došlić, Bipartivity of fullerene graphs and fullerene stability. Chemical Physics Letters 412 (2005) 336-340.

[7] Ernesto Estrada and Juan A. Rodríguez-Velázquez, Subgraph centrality in complex networks. Physical Review E 71, 056103 (2005) 9 pages.

[8] Ernesto Estrada and Juan A. Rodríguez-Velázquez, Spectral measures of bipartivity in complex networks. Physical Review E 72, 046105 (2005) 6 pages.

[9] Ernesto Estrada and Juan A. Rodríguez-Velázquez, Subgraph Centrality and clustering in complex hyper-networks. Physica A 364 (2006) 581594.

[10] L. C. Freeman, Centrality in social networks: I. Conceptual clarification. Social Networks 1 (1979) 215-239.

[11] L. C. Freeman, A Set of Measures of Centrality Based on Betweenness. Sociometry 40 (6) (1977) 35-41. 
[12] M. E. J. Newman, Analysis of weighted networks. Physical Review E 70 056131 (2004) 9 pages.

[13] J. A. Rodríguez, On the Laplacian spectrum and walk-regular hypergraphs. Linear and Multilinear Algebra 51 (2003) 285-297.

[14] D. J. Watts, Small World: The Dynamics of Networks Between Order and Randomness. Princeton Univ. Press, Princeton, NJ, 1999. 